\title{
Genehmigungsverfahren für klassische Gewebe- zubereitungen gemäß § 21a Arzneimittelgesetz (AMG)
}

\author{
Dagmar Schilling-Leiß Antonia W. Godehardt Jürgen Scherer Klaus Cichutek Ralf R. Tönjes
}

Abteilung Medizinische Biotechnologie, Paul-Ehrlich-Institut, Langen, Deutschland

\author{
Schlüsselwörter \\ Gewebe - Gewebezubereitungen - Genehmigung • \\ Gewebegesetz
}

\section{Zusammenfassung}

Am 1. August 2007 trat in Deutschland das Gewebegesetz in Kraft, welches unter anderem Regelungen hinsichtlich der Qualität und Sicherheit von Gewebezubereitungen (GWZ) trifft. Klassische GWZ sind Arzneimittel für die Anwendung am Menschen, a) die menschliche Gewebe und Zellen enthalten oder ausgehend von diesen hergestellt werden, b) deren wesentliche Be- oder Verarbeitungsverfahren in der EU hinreichend bekannt bzw. neu, aber mit bekannten Verfahren vergleichbar sind, und c) deren Wirkungen und Nebenwirkungen aus dem wissenschaftlichen Erkenntnismaterial ersichtlich sind. Dazu gehören z.B. Herzklappen, Corneae, muskuloskelettale GWZ, Gefäße oder Stammzellen aus Knochenmark. Wenn bei einer GWZ die oben genannten Voraussetzungen erfüllt sind und diese nicht mit ionisierenden Strahlen behandelt wird, ist für das Inverkehrbringen eine Genehmigung gemäß § 21a Abs. 1 Arzneimittelgesetz (AMG) durch das Paul-Ehrlich-Institut erforderlich. Zur Erleichterung der Antragstellung für die Genehmigung stehen Formulare auf der Internetseite des Paul-EhrlichInstituts zur Verfügung. Schwerwiegende unerwünschte Reaktionen und schwerwiegende Zwischenfälle gemäß § 63c AMG müssen von Gewebeeinrichtungen unverzüglich an die zuständige Bundesbehörde gemeldet werden. Des Weiteren besteht für Gewebeeinrichtungen eine Meldepflicht gemäß $\S 8 d$ Abs. 3 Transplantationsgesetz (TPG) zur Erfassung der verarbeiteten Gewebe, die in Form eines jährlichen Berichts bis zum 1. März des folgenden Jahres an das Paul-Ehrlich-Institut erfolgt.

\author{
Key Words \\ Tissues - Tissue preparations - Authorization . \\ Tissue Law
}

\section{Summary \\ Authorization Procedures for Classical Tissue Preparations according to Section 21a German Medicinal Products Act (Arzneimittelgesetz; AMG)}

The German Tissue Law which mainly regulates the quality and safety of tissue preparations (Gewebezubereitungen; GWZ) came into effect on August 1, 2007. Classical tissue preparations are human medicinal products that fulfil the following prerequisites: i) They are prepared starting from human tissues and cells or they contain human tissues and cells; ii) the essential processing procedures are sufficiently well known or new, but comparable with known procedures; iii) the effects and side effects are known and evident from scientific data. Examples of classical tissue preparations are heart valves, corneae, musculoskeletal tissue preparations, vessels or stem cells from bone marrow. If the prerequisites listed above are fulfilled and if the given tissue preparation has not been treated using ionizing radiation, an authorization according to Section 21a AMG by the Paul Ehrlich Institute is required prior to placing the tissue preparation on the market. In order to facilitate applying for the authorization of tissue preparations, the Paul Ehrlich Institute has published standard forms on its internet website. Tissue establishments immediately have to notify the competent authority of any serious adverse events and serious incidents according to Section 63c AMG. In addition, according to Section 8d (3) of the German Transplantation Law (TPG), tissue establishments are obliged to report processed tissues for data recording purposes. Data have to be submitted via an annual report on standard forms to the Paul Ehrlich Institute until 1st of March of the following year.

\begin{tabular}{ll}
\hline KARGER & $\oplus$ 2008 S. Karger GmbH, Freiburg \\
Fax +49 7614520714 & Accessible online at: \\
Information@Karger.de & www.karger.com/tmh
\end{tabular}

Prof. Dr. Ralf R. Tönjes

Abteilung Medizinische Biotechnologie, Paul-Ehrlich-Institu

Paul-Ehrlich-Strasse 51-59, 63225 Langen, Deutschland

toera@pei.de 


\section{Einleitung}

Am 1. August 2007 trat in Deutschland das Gewebegesetz in Kraft. Mit diesem Gesetz wurde die Richtlinie 2004/23/EC der Europäischen Union (EU) in deutsches Recht überführt, während die Richtlinien 2006/17/EC und 2006/86/EC mit der Verordnung zur Änderung der Arzneimittel- und Wirkstoff-Herstellungsverordnung (AMWHV) und der Transplantationsgesetz-Gewebeverordnung (TPG-GewV) in deutsches Recht überführt wurden. Das Gewebegesetz trifft unter anderem Regelungen hinsichtlich der Qualität und Sicherheit von Gewebezubereitungen (GWZ), d.h. von Arzneimitteln, die menschliche Gewebe im Sinne des § 1a Nr. 4 TPG enthalten oder aus solchen hergestellt werden.

Gemäß § 1a Nr. 4 TPG in Verbindung mit § 1a Nr. 1 TPG sind Gewebe alle menschlichen Gewebe und Zellen inklusive Haut, mit Ausnahme der Organe. Mit der 12. Arzneimittelgesetz(AMG)-Novelle, die am 6. August 2004 in Kraft trat, wurde das Paul-Ehrlich-Institut für GWZ zuständig (§ 77 Abs. 2 AMG) und damit verbunden für die Zulassung und die Genehmigung von GWZ, die Pharmakovigilanz, die Genehmigung der klinischen Prüfung und das Meldewesen gemäß $\S 8 \mathrm{~d}$ Abs. 3 TPG für diese Arzneimittel.

Klassische GWZ (siehe «Einteilung der Genehmigungsanträge für klassische Gewebezubereitungen») umfassen z.B. Herzklappen, Gefäße, Corneae und muskuloskelettale GWZ. Von den klassischen GWZ werden autologe und gerichtet allogene Stammzellzubereitungen aus peripherem Blut bzw. Nabelschnurblut sowie Arzneimittel für neuartige Therapien unterschieden (siehe «Beispiele für Ausnahmen vom Gewebegesetz und besondere Gewebezubereitungen»). Für das Inverkehrbringen einer klassischen GWZ in Deutschland wird entweder eine Genehmigung gemäß § 21a Abs. 1 AMG, eine Bescheinigung gemäß § 21a Abs. 9 AMG (siehe «Anforderungen für eine Genehmigung gemäß § 21a AMG») oder eine Zulassung gemäß $§ 21 \mathrm{ff}$. AMG durch das Paul-Ehrlich-Institut benötigt. Jede der drei folgenden Voraussetzungen gemäß $§ 21 \mathrm{a}$ Abs. 1 AMG muss erfüllt sein, damit eine GWZ genehmigungs- und nicht zulassungspflichtig ist: 1) die Herstellung der GWZ erfolgt nicht unter Anwendung eines oder mehrerer industrieller Verfahren, 2) die wesentlichen Be- oder Verarbeitungsverfahren sind in der EU hinreichend bekannt bzw. die Be- oder Verarbeitungsverfahren sind neu, aber mit bekannten Verfahren vergleichbar und 3) die Wirkungen und Nebenwirkungen sind aus dem wissenschaftlichen Erkenntnismaterial ersichtlich. Zudem darf die GWZ nicht mit ionisierenden Strahlen behandelt werden (siehe «Beispiele für Ausnahmen vom Gewebegesetz und besondere Gewebezubereitungen»), anderenfalls ergibt sich eine Zulassungspflicht.

Für die Entnahme von Geweben sowie die zugehörigen Laboruntersuchungen bedarf es einer Erlaubnis gemäß $\S 20 \mathrm{~b}$ AMG, die von der zuständigen Landesbehörde erteilt wird. Bei der Erlaubniserteilung kann das Paul-Ehrlich-Institut von der zuständigen Landesbehörde beteiligt werden. Für die
Konservierung, Lagerung sowie die Be- oder Verarbeitung von Geweben wird eine Erlaubnis gemäß § 20c AMG bzw. eine Herstellungserlaubnis gemäß $§ 13$ AMG benötigt, die die zuständige Behörde des Landes im Benehmen mit dem PaulEhrlich-Institut erteilt. Unter Umständen kann die Be- und Verarbeitungserlaubnis eine oder mehrere Entnahmestellen beinhalten. Auf die Voraussetzungen für die Erlaubnisse gemäß § 20b und 20c AMG und für eine Zulassung gemäß § 21ff AMG sowie für die Produkteinfuhr wird hier nicht näher eingegangen. Im Folgenden wird die Verwendung der Antragsformulare (www.pei.de/cln_115/nn_748690/DE/infos/pu/040genehmigung-21a-amg/01-gewebe/01-antrag-genehmigung/antrag-genehmigung-node.html?_nnn = true), die das Paul-Ehrlich-Institut zur Unterstützung der Antragsteller für die Genehmigung einer klassischen GWZ gemäß § 21a Abs. 1 AMG zur Verfügung gestellt hat, an einigen Beispielen erläutert (siehe «Antragsformulare für die Genehmigung von Gewebezubereitungen gemäß $\S 21 \mathrm{a}$ Abs. 1 AMG»). Für die Antragstellung von Stammzellen aus dem menschlichen Knochenmark empfiehlt das Paul-Ehrlich-Institut die Antragsformulare (www.pei.de/cln_108/nn_748690/DE/infos/pu/040-genehmigung-21a-amg/02-stammzellen/ stammzellzubereitungennode.html? _nnn=true), die für die Stammzellzubereitungen der autologen und gerichtet allogenen Anwendung vorgesehenen sind.

\section{Einteilung der Genehmigungsanträge für klassische Gewebezubereitungen}

Klassische GWZ sind Arzneimittel gemäß Definition in $\S 2$ Abs. 1 AMG, da es sich um Stoffe und Zubereitungen aus Stoffen handelt, die dazu bestimmt sind, durch Anwendung am oder im menschlichen Körper Krankheiten, Leiden, Körperschäden oder krankhafte Beschwerden zu heilen, zu lindern oder zu verhüten. Klassische GWZ sind Arzneimittel, a) die menschliche Gewebe oder Zellen enthalten oder ausgehend von diesen hergestellt werden, b) deren wesentliche Be- oder Verarbeitungsverfahren in der EU hinreichend bekannt bzw. neu, aber mit bekannten Verfahren vergleichbar und c) deren Wirkungen und Nebenwirkungen aus dem wissenschaftlichen Erkenntnismaterial ersichtlich sind. Für das Inverkehrbringen ist eine Genehmigung gemäß $§ 21 \mathrm{a}$ Abs. 1 AMG durch das Paul-Ehrlich-Institut erforderlich. Durch die Genehmigung wird das Verbot, gemäß $§ 17$ TPG mit Geweben zu handeln, nicht aufgehoben.

GWZ, die in Verkehr gebracht werden sollen, sind gemäß $\S 21$ a Abs. 1 AMG genehmigungs-, und nicht zulassungspflichtig, wenn bei ihrer Be- oder Verarbeitung kein industrielles Verfahren angewendet und keine Behandlung mit ionisierenden Strahlen vorgenommen wird (siehe «Beispiele für Ausnahmen vom Gewebegesetz und besondere Gewebezubereitungen»).
Schilling-Leiß/Godehardt/Scherer/ Cichutek/Tönjes 


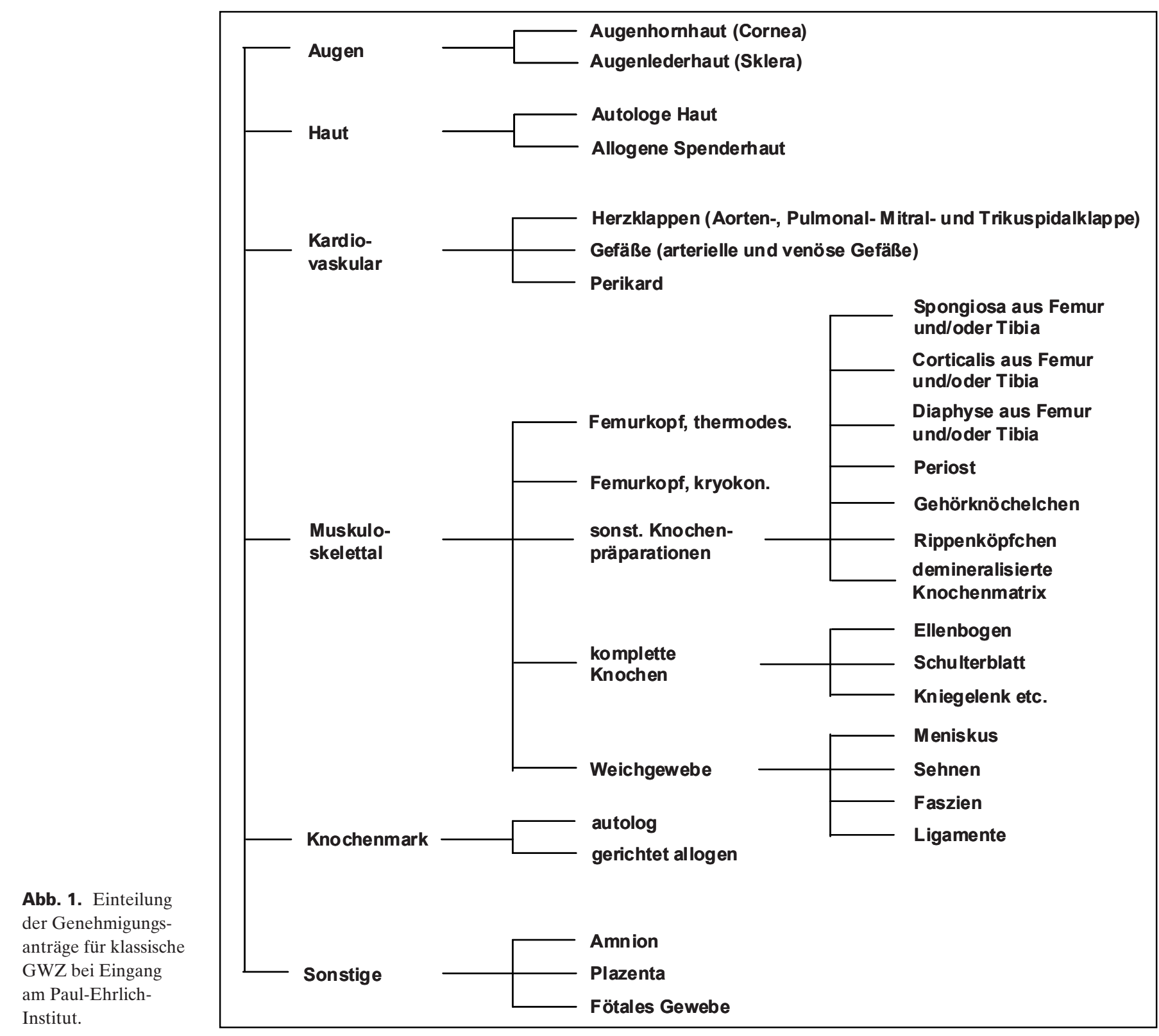

Das Paul-Ehrlich-Institut unterteilt eingehende Genehmigungsanträge für klassische GWZ zur Zeit in sechs Hauptgruppen und weitere Untergruppen (Abb. 1). Die Hauptgruppen beinhalten GWZ aus dem Auge, der Haut, dem kardiovaskulären System, dem Knochenmark sowie muskuloskelettale GWZ, komplettiert durch eine heterogene Gruppe sonstiger GWZ. Mit Ausnahme von Stammzellen aus dem Knochenmark (siehe unten) ist den GWZ jeder Hauptgruppe zu eigen, dass die Anforderungen an die Genehmigungen vergleichbar sind und die zur Be- und Verarbeitung entnommenen Gewebe oder Zellen weitgehend aus dem gleichen anatomischen Bereich stammen.

Die Hauptgruppen werden in weitere Untergruppen aufgeteilt. Genehmigungsanträge für Aorten-, Pulmonal-, Mitralund Trikuspidalklappen wurden z.B. aufgrund gleicher Verfahren der Vorbereitung, Herstellung und vergleichbarer Kri- terien der Qualitätsbeurteilung sowie gleicher Indikation in der Untergruppe «Herzklappen» zusammengefasst. Im Gegensatz dazu begründet sich bei der Untergruppe der «ganzen Knochen» eine weitere Aufteilung der §-21a-AMG-Genehmigungsanträge in GWZ aus z.B. Ellenbogengelenk, Schulterblatt oder Kniegelenk durch den unterschiedlichen anatomischen Ursprung in Verbindung mit unterschiedlichen medizinischen Verwendungszwecken und Funktionalität.

Bei Genehmigungsanträgen für GWZ, die Stammzellen aus dem menschlichen Knochenmark enthalten, wird zwischen der autologen und der gerichtet allogenen Verwendung unterschieden. Die zuletzt genannten Genehmigungsanträge werden mit §-21a-Genehmigungsanträgen für Blutstammzellen aus peripherem menschlichen Blut oder Nabelschnurblut als Stammzellzubereitungen zusammengefasst und gemeinsam behandelt, weil hier vergleichbare Anforderungen zugrunde 
Tab. 1. Beispiele für Ausnahmen von der Genehmigungspflicht gemäß § 21a AMG bzw. vom Anwendungsbereich des AMG

\begin{tabular}{|c|c|}
\hline Gewebe / GWZ & Begründung/Ausnahme \\
\hline $\begin{array}{l}\text { Gefäße bei Organtransplantation, die bei Transplantation } \\
\text { eines Organs mit diesem verbunden sind }\end{array}$ & sind Organe gemäß $§ 1$ a Nr. 1 TPG und keine Gewebe oder GWZ \\
\hline Nebenschilddrüsen & sind Organe gemäß $\S 1$ a Nr. 1 TPG \\
\hline Schädelkalotte & $\begin{array}{l}\text { vom AMG-Anwendungsbereich ausgenommen gemäß § 4a Nr. } 4 \text { AMG } \\
\text { (laut Bundesdrucksache 16/5443) }\end{array}$ \\
\hline Samen und Eizellen & keine GWZ (gemäß § 4 Abs. 30 AMG) \\
\hline Hoden- und Nebenhodengewebe & keine GWZ, falls zur Gewinnung von Samenzellen verwendet \\
\hline Mit ionisierenden Strahlen behandelte Gewebe & zulassungspflichtig nach $\S 1$ Abs. 2 Nr. 4 AMRadV in Verbindung mit $\S 21 \mathrm{ff}$ AMG \\
\hline GWZ, die zu den Arzneimitteln für neuartige Therapien zählen & $\begin{array}{l}\text { zulassungspflichtig gemäß VO (EG) Nr. 726/2004, zum Teil mit Übergangsfristen } \\
\text { (zentrales europäisches Zulassungsverfahren) }\end{array}$ \\
\hline
\end{tabular}

gelegt werden und organisatorische Überlegungen des PaulEhrlich-Instituts zum Tragen kommen. Für die Beantragung der Genehmigung der Stammzellzubereitungen gemäß § 21a Abs. 1 AMG stellt das Paul-Ehrlich-Institut gesonderte Antragsformulare zur Verfügung (siehe Hinweis in «Einleitung»).

\section{Beispiele für Ausnahmen vom Gewebegesetz und besondere Gewebezubereitungen}

Einige Gewebe und Zellen gelten im Sinne des Gewebegesetzes nicht als Gewebe oder fallen nicht unter die Definition einer GWZ. Zusätzlich findet das AMG in bestimmten Fällen keine Anwendung auf GWZ (Tab. 1).

\section{Gewebe, die zum gleichen Zweck wie Organe übertragen werden}

Gemäß § 1a Nr. 1 TPG gelten Gewebe, die zum gleichen Zweck wie Organe übertragen werden, als Organe und werden von den für GWZ im Gewebegesetz getroffenen Regelungen nicht erfasst. Darunter fallen z.B. Gefäße, die unmittelbar mit einem Organ verbunden und für eine erfolgreiche Transplantation des Organs erforderlich sind. Sie bilden eine funktionelle Einheit mit dem Organ und fallen somit unter den Organbegriff. Das gilt nach Ansicht des Paul-Ehrlich-Instituts gleichermaßen für solche peripheren Gefäße, die für eine erfolgreiche Übertragung eines vom selben Spender stammenden Organs erforderlich sind und gemeinsam mit dem Organ für dessen Fähigkeit zum Vollzug physiologischer Funktionen im Sinne des $\S 1$ a Nr. 1 TPG transplantiert werden.

Das Paul-Ehrlich-Institut hat auf Nachfrage verschiedener zuständiger Landesbehörden auch seine Einschätzung geäußert, dass Nebenschilddrüsen ebenfalls als Organe gemäß $§ 1$ a Nr. 1 TPG eingestuft werden können, weil sie als funktionelle Einheit in Bezug auf Struktur, Blutgefäßversorgung und Fähigkeit zum Vollzug physiologischer Funktionen anzusehen sind. Die auch als Epithelkörperchen bezeichneten Nebenschild- drüsen werden bei sekundärem Hyperparathyroidismus, bedingt z.B. durch eine chronische Niereninsuffizienz, aber auch bei Schilddrüsenoperationen in den Unterarm autotransplantiert und dadurch endokrin aktiv.

Ausnahmen vom Anwendungsbereich des AMG gemäß $\S \S 4 a$ Nr. 3 und Nr. 4 AMG

Die Landesbehörden entscheiden im Rahmen der Überwachung oder im Zusammenhang mit Antragsvorhaben gemäß $\S \S 20 \mathrm{~b}$ und 20c AMG über die Anwendbarkeit der Ausnahmeregelungen in $\S \S 4 \mathrm{a}$ Nr. 3 und Nr. 4 AMG. Generell findet das AMG keine Anwendung auf Arzneimittel, die gemäß $\S 4$ a Nr. 3 AMG «unter der unmittelbaren fachlichen Verantwortung des anwendenden Arztes hergestellt worden sind». Ebenso fallen gemäß § 4a Nr. 4 AMG «Gewebe, die innerhalb eines Behandlungsvorgangs einer Person entnommen werden, um auf diese rückübertragen zu werden» unter die Ausnahmeregelung des AMG. Laut Beschlussempfehlung des Deutschen Bundestages vom 23. Mai 2007 [1] trifft letzteres z.B. bei einer medizinisch erforderlichen Entfernung der Schädelkalotte zu, die erst nach Abschluss der anschließenden medizinischen Behandlung rückübertragen wird.

Samen- und Eizellen sowie Hoden und Nebenhodengewebe In $\$ 4$ Abs. 30 AMG ist festgelegt, dass menschliche Samenund Eizellen, einschließlich imprägnierter Eizellen (Keimzellen), und Embryonen keine GWZ sind. Daher besteht für sie keine Zulassungs- oder Genehmigungspflicht, die für das Inverkehrbringen von GWZ notwendig ist. Nach Ansicht des Paul-Ehrlich-Instituts ist diese Ausnahmeregelung auch auf Hoden- und Nebenhodengewebe anzuwenden, solange dieses Gewebe ausschließlich im Sinne der Reproduktionsmedizin zur Gewinnung von Samenzellen angewendet wird.

\section{Bestrahlte GWZ}

Das generelle Verkehrsverbot gemäß $§ 7$ Abs. 1 AMG für mit ionisierenden Strahlen behandelte Arzneimittel kann gemäß $\S 1$ Abs. 2 Nr. 4 der Verordnung über radioaktive oder mit 
ionisierenden Strahlen behandelte Arzneimittel (AMRadV) für GWZ, die zur Verminderung der Keimzahl bestrahlt werden, durch eine Zulassung nach § 25 Abs. 1 AMG aufgehoben werden. Da eine Genehmigung gemäß § 21a AMG dieses Verkehrsverbot nicht aufhebt, ist derzeit für das Inverkehrbringen einer mit ionisierenden Strahlen behandelten GWZ eine Zulassung gemäß $§ 25$ Abs. 1 AMG erforderlich. Das PaulEhrlich-Institut hat vorgeschlagen, die AMRadV zu ändern, so dass neben der Zulassung auch eine Genehmigung gemäß $\S 21 \mathrm{a}$ AMG das oben genannte generelle Verkehrsverbot gemäß $§ 7$ Abs. 1 AMG für mit ionisierenden Strahlen behandelte Arzneimittel aufheben kann.

\section{GWZ, die zu den Arzneimitteln für neuartige Therapien gehören}

Neben den sogenannten «klassischen» GWZ gibt es GWZ, die zu den Arzneimitteln für neuartige Therapien (advanced therapy medicinal products; ATMP) zählen. Diese werden unterteilt in 1) Gentherapie-Arzneimittel, 2) humane somatische Zelltherapie-Arzneimittel und 3) «Tissue engineering»-Arzneimittel. Die ersten beiden Arzneimittelgruppen werden in Teil IV des Anhangs I der Richtlinie 2001/83/EG definiert. Die Arzneimittel der dritten Gruppe, die auch als biotechnologisch bearbeitete Gewebeprodukte bezeichnet werden, sind in der Verordnung (EG) Nr. 1394/2007 definiert. Für ATMP wird das zentrale europäische Zulassungsverfahren gemäß Verordnung (EG) Nr. 726/2004 angewandt, wobei die Übergangsfristen gemäß Verordnung (EG) Nr. 1394/2007 gelten. Weiterhin können bei einer GWZ die im Anhang der Verordnung (EG) Nr. 726/2004 genannten Voraussetzungen zutreffen. Gemäß dieses Anhangs sind GWZ, die einen neuen Wirkstoff enthalten, ebenfalls über das von der Europäischen Arzneimittelagentur (European Medicines Agency; EMEA) koordinierte zentrale europäische Verfahren zulassungspflichtig. Das gilt für Wirkstoffe, die spätestens seit 20. Mai 2008 noch nicht in der Europäischen Gemeinschaft genehmigt waren und deren therapeutische Indikationen das erworbene Immundefizienz-Syndrom, Krebs, neurodegenerative Erkrankungen, Diabetes, Autoimmunerkrankungen und andere Immunschwächen oder Viruserkrankungen sind. Eine diese Fälle erläuternde Schrift des Committee for Medicinal Products for Human Use (CHMP) steht bei der EMEA zur Verfügung (www.emea.europa.eu/pdfs/human/regaffair/12194407en.pdf). Eine Ausnahme von der Zulassungspflicht besteht in Deutschland gemäß $\S 21$ Abs. 2 Nr. 1a AMG derzeit bis Ablauf der in der Verordnung (EG) Nr. 1394/2007 genannten Übergangsfrist unter anderem für Arzneimittel, die autologe Körperzellen enthalten und die somatische Zelltherapeutika gemäß $§ 4$ Abs. 20 AMG sind und im Rahmen der Gewebezüchtung zur Geweberegeneration aufbereitet und vermehrt werden. Für das Inverkehrbringen dieser Arzneimittel wird grundsätzlich eine Herstellungserlaubnis gemäß $§ 13$ Abs. 1 AMG und eine Entnahmeerlaubnis gemäß § 20b AMG benötigt. Gemäß Verordnung (EG) Nr. 1394/2007 dürfen diese
Arzneimittel in den Jahren 2012 bzw. 2013 nicht mehr ohne Zulassung in den Verkehr gebracht werden.

\section{Anforderungen für eine Genehmigung von Gewebe- zubereitungen gemäß $\S 21 a$ AMG}

Die drei Voraussetzungen für die Genehmigungspflicht einer klassischen GWZ gemäß $§ 21$ a Abs. 1 AMG sind in der Einleitung bereits erwähnt. Dazu gehört, dass bei der Be- oder Verarbeitung der GWZ keine industriellen Verfahren zum Einsatz kommen, z.B. solche, bei denen routinemäßig gleichartige festgelegte Prozesse durchgeführt werden. Ausweislich der Begründung zu Artikel 2 Nr. 11b des Gewebegesetzes liegt eine industrielle Herstellung dann vor, wenn für die Beoder Verarbeitung des Gewebes anspruchsvolle technische oder aufwändige maschinelle Verfahren zum Einsatz kommen [1]. Auch eine Herstellung in größerem Umfang oder auf Vorrat für einen nicht bekannten Abnehmerkreis sind Indizien für eine industrielle Herstellung [2].

Bei der Antragstellung ist zu belegen, dass die in $\S 21 \mathrm{a}$ Abs. 1 AMG genannten Voraussetzungen zutreffen. Die eingereichten Unterlagen müssen eine Beurteilung des Arzneimittels hinsichtlich Funktionalität und Unbedenklichkeit zulassen (siehe «Antragsformulare für die Genehmigung von Gewebezubereitungen gemäß § 21a Abs. 1 AMG»).

Bei erstmaligem Verbringen einer GWZ aus einem anderen EU-Mitgliedstaat oder einem anderen Vertragsstaat des Abkommens über den Europäischen Wirtschaftsraum (EWR) ist eine Bescheinigung des Paul-Ehrlich-Instituts gemäß $\S 21 \mathrm{a}$ Abs. 9 AMG erforderlich. Hierbei hat das Paul-Ehrlich-Institut zu prüfen, ob die Be- oder Verarbeitungsverfahren sowie die quantitativen und qualitativen Kriterien der GWZ den Anforderungen des AMG und seinen Verordnungen an die Entnahme, die Verarbeitung, die Spenderauswahlverfahren und die Laboruntersuchungen entsprechen bzw. diesen gleichwertig sind. Im einfachsten Falle können dazu die rechtlichen Anforderungen in Deutschland mit den rechtlichen Anforderungen des Staates, aus dem erstmalig eingeführt werden soll, verglichen werden. Vom Paul-Ehrlich-Institut wird vorausgesetzt, dass mit dem Antrag gemäß $§ 21$ Abs. 9 AMG ein Nachweis der Genehmigung der GWZ durch den EU-Mitgliedstaat bzw. den EWR-Vertragsstaat vorgelegt wird, die hinsichtlich der in $\S 21 \mathrm{a}$ Abs. 9 AMG genannten Anforderungen mit der Genehmigung gemäß $§ 21$ a Abs. 1 AMG vergleichbar ist. Bei negativ ausfallendem Vergleich oder wenn die Richtlinie 2004/23/EC noch nicht transformiert wurde, kann die oben genannte Bescheinigung nicht ausgestellt werden. In diesen Fällen kann ein Antrag gemäß § 21a Abs. 1 AMG gestellt werden. Da momentan eine Reihe von EU-Mitgliedstaaten die Richtlinie 2004/23/EG nicht transformiert haben, empfahl das Paul-Ehrlich-Institut zum Stichtag 1. Februar 2008 eine Antragstellung gemäß § 21a Abs. 1 AMG unter Verwendung der auf der Internetseite (www.pei.de) für eine Genehmigung 
Abb. 2. Schematische Darstellung des Aufbaus eines Genehmigungsantrags für klassische GWZ. Die Module 1-5 sind an die Erfordernisse des § 21a AMG angepasste Versionen des international vorgeschriebenen CTD (Common Technical Document), die als G-CTD (Genehmigungs-CTD) bezeichnet werden.

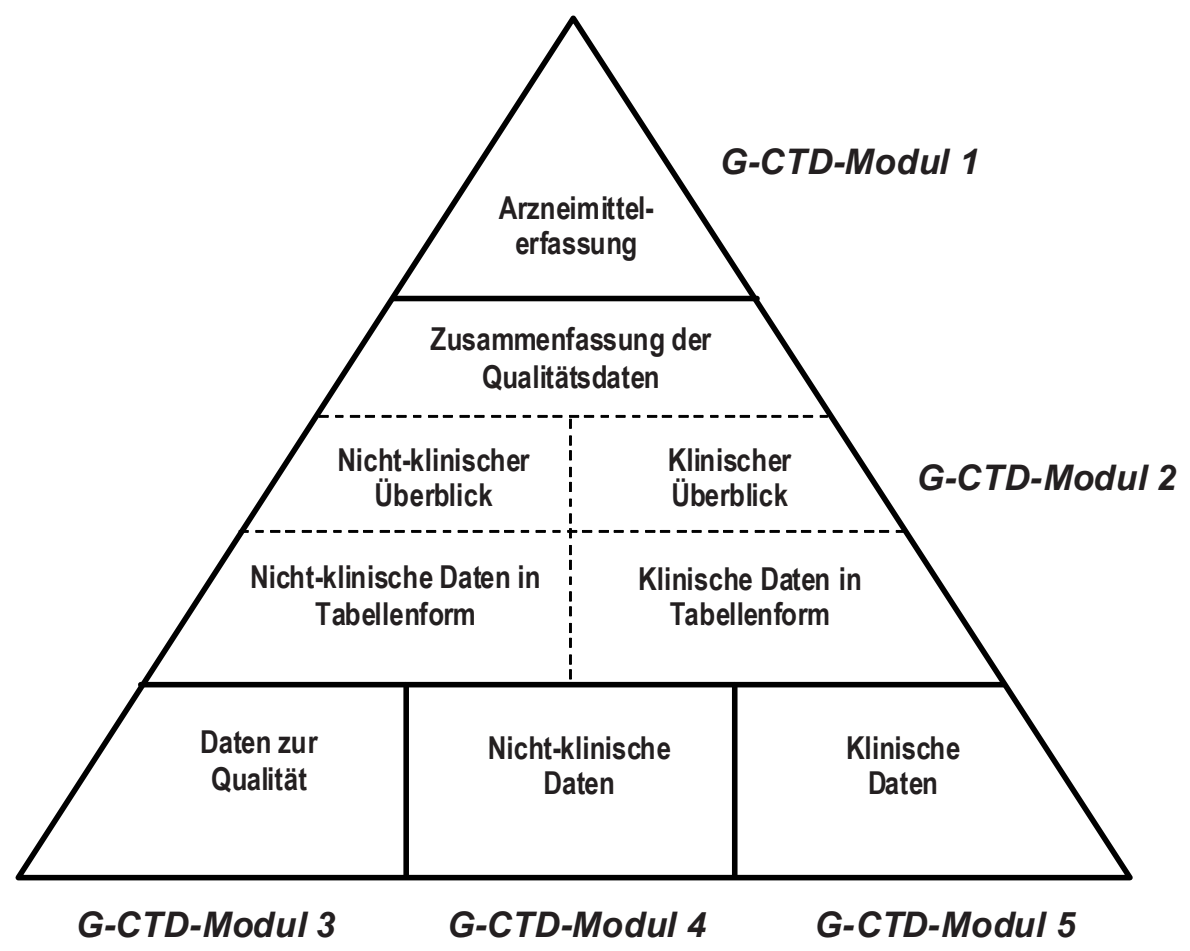

hergestellte Arzneimittel ist ein eigener Genehmigungsantrag zu stellen. Eine GWZ wird typischerweise nach einem festgelegten Verfahren mit vergleichbarem Ausgangs- bzw. Spendermaterial und Hilfsstoffen be- oder verarbeitet, ist, wie z.B. ein Knochenwürfel, durch eine definierte Form, Größe oder Qualität gekennzeichnet und soll für eine definierte, abgegrenzte Indikation eingesetzt werden.

Die GWZ, für die ein Antrag eingereicht wurde, muss einen Produktnamen erhalten. Der Produktname soll Hinweise auf die Herkunft, die Art des Gewebes und dessen Spezifikation enthalten, um eine Abgrenzung zu anderen Arzneimitteln mit ähnlichen Charakteristika sicherzustellen. Beispiele für eine solche Namensgebung wären «Human-Cornea-Mediumkultiviert-Freiburg», «Human-Tibia-Kryokonserviert-Köln» oder «Human-Femurkopf-Thermodesinfiziert-KryokonserviertBerlin». Die Bezeichnung der beantragten GWZ, d.h. der Produktname, muss eindeutig aus dem Anschreiben an das PaulEhrlich-Institut sowie den eingereichten Unterlagen hervorgehen. Wie bereits beschrieben, können unter bestimmten Voraussetzungen vergleichbare GWZ, die z.B. mit dem gleichen Verfahren hergestellt werden, in einem Genehmigungsantrag unter der gleichen Produktbezeichnung zusammengefasst werden (siehe «Einteilung der Genehmigungsanträge für klassischen Gewebezubereitungen»). Ein Beispiel für eine solche Zusammenfassung sind die Aorten- und Pulmonalklappen, für die als humane Homografts nur ein Antrag zu stellen wäre. Die Zusammenfassung ist jedoch im Rahmen der Antragstellung individuell in Rücksprache mit dem Antragsteller zu bewerten und bedarf der Zustimmung des Paul-Ehrlich-Instituts. 
Aufgrund der Vielzahl unterschiedlicher GWZ wurde bei dem G-CTD-Antragsformular darauf verzichtet, gewebespezifische Anpassungen vorzunehmen. Es kann daher für alle klassischen GWZ (Abb. 1) gleichermaßen verwendet werden. Dies hat zur Folge, dass die vorgegebenen Gliederungspunkte nicht für alle Produkte (GWZ) gleichermaßen zutreffen. Aus diesem Grund ist im Hinblick auf die Antragsstellung vorgesehen, die für das Produkt nicht relevanten Gliederungspunkte als «nicht zutreffend» zu vermerken. Sollte es erforderlich sein, gibt es in den im Formular vorgegebenen Abschnitten die Möglichkeit, neue Gliederungspunkte einzufügen. Das Einfügen oder Streichen von Gliederungspunkten ist deutlich zu machen und kurz zu begründen.

Der Antrag auf Genehmigung (G-CTD) ist in fünf Module unterteilt, in denen die vorhandenen Daten zu der GWZ gezielt abgefragt werden (Abb. 2). Ein Antrag gemäß § 21a Abs. 1 AMG beinhaltet allgemeine Daten zur Arzneimittelerfassung in Modul 1, Informationen zur Qualität sowie Zusammenfassungen und bewertende Diskussionen nichtklinischer und klinischer Daten in Modul 2 und eine Darstellung der Verfahrensabläufe von der Gewinnung/Entnahme über die Verarbeitung bis hin zum fertigen Arzneimittel in Modul 3. Beschriebene Daten und Referenzen sollen in den Modulen 4-5 zur Verfügung gestellt werden. Die einzelnen G-CTDModule werden im Folgenden kurz beschrieben und die darin erforderlichen Angaben anhand von Fallbeispielen erläutert.

\section{G-CTD-Modul 1: Arzneimittelerfassung}

Modul 1 des Genehmigungsantrags dient der ersten Erfassung des eingereichten Arzneimittels. Hier sollen unter anderem das Anschreiben eingefügt, die vorgegebene Inhaltsübersicht des Antrags mit Seitenzahlen versehen, das Antragsformular (Modul 1.2) ausgefüllt und die erbetenen Unterlagen wie z.B. die Kurzbeschreibung der Be- oder Verarbeitung und ein Flussdiagramm zur Darstellung der Gewinnung, der Verarbeitungs- und Verfahrensschritte und Prüfung, als nummerierte Anlagen beigefügt werden. Eine tabellarische Übersicht der geforderten Anlagen ist im Antragsformular enthalten. In Modul 1.3 (Produktinformation) sind der Wortlaut der Gebrauchs- und Fachinformation sowie der Wortlaut für die Behältnisbeschriftung und gegebenenfalls der äußeren Umhüllung einzufügen.

In Modul 1 soll entsprechend des Anschreibens die Bezeichnung der zu genehmigenden GWZ angegeben werden, um diese von anderen genehmigten oder zugelassenen GWZ eindeutig zu unterscheiden. Beispiele für eine solche Bezeichnung wurden bereits im vorhergehenden Abschnitt aufgeführt. Auch die beabsichtigte Darreichungsform der GWZ, z.B. als feste Substanz, Gel oder Paste, in Form einer Lösung oder als unveränderte feste Struktur, ist anzugeben. Bei muskuloskelettalen GWZ aus Knochen könnte dies die Beschreibung der Form sein (Würfel, Stifte oder Keile). Weitere Beschreibungen können die Struktur (Pulver, Granula oder Knochenkit) betreffen.

\section{G-CTD-Modul 2: Zusammenfassungen}

G-CTD-Modul 2 dient der Zusammenfassung, Diskussion und Bewertung des vorhandenen und in weiteren Modulen dargelegten Datenmaterials. In den Modulen 2.3-2.5 sollen die vorliegenden und bereits in anderen Antragsmodulen im Detail dargelegten Daten zur Qualität der GWZ (Modul 2.3: Zusammenfassung der Qualitätsdaten), Ergebnisse von nichtklinischen (Modul 2.4: Nichtklinischer Überblick zum Nachweis der Funktionalität und Risiken der GWZ) und klinischen Prüfungen (Modul 2.5: Klinischer Überblick zum Nachweis der Funktionalität und Risiken der GWZ) zur Funktionalität und Hinweise zu Risiken vom Antragsteller kurz zusammengefasst, diskutiert und bewertet werden. Aus der Diskussion soll die Einschätzung des Antragstellers auf Basis der im gesamten Antrag vorgelegten Daten hervorgehen, ob die GWZ die gemäß $\S 21$ a Abs. 6 Nr. 2 und Nr. 3 geforderten Kriterien erfüllt. Detailerläuterungen sind in den oben genannten Modulteilen enthalten.

Modul 2.3 fasst die Qualitätsdaten zusammen und sollte hinreichende Informationen zu jedem Abschnitt der in G-CTDModul 3 dargestellten Daten zur Qualität enthalten. Die Beoder Verarbeitungsverfahren sowie die Ergebnisse von mikrobiologischen, chemischen und physikalischen Prüfungen der GWZ und die zur Ermittlung angewandten Methoden sind auch in der hier anzugebenden Zusammenfassung kurz und übersichtlich darzustellen.

In Modul 2.4 sollen die nichtklinischen Ergebnisse zur Funktionalität und zu den Risiken der GWZ erläutert werden. Schwerpunkte in der nichtklinischen Charakterisierung von GWZ können unter anderem die Immunogenität, die lokale Toleranz, die biomechanische Stabilität oder andere pharmakologische Eigenschaften wie die Pharmakodynamik, Pharmakokinetik und Toxikologie sein. Wenn möglich, soll anhand von wissenschaftlichem Erkenntnismaterial oder Studien das Wirkprofil, die Dosis-Wirkungs-Beziehung oder der Wirkmechanismus der verabreichten GWZ diskutiert und bewertet werden. Das Wirkprofil bezieht sich in diesem Fall auf die Art und den Ort der Wirkung. Es soll erläutert werden, ob Organe oder andere Strukturen bzw. biologische Funktionen beeinflusst werden. Ein Beispiel für Angaben zur Dosis-WirkungsBeziehung sind im Falle von demineralisierter Knochenmatrix die Definition der wirksamen Konzentration des eingesetzten Knochenmaterials im Hinblick auf den intendierten Wirkmechanismus und die Indikation. Im Falle der Herzklappen kann dies der Anteil von lebendem Spendergewebe wie z.B. Fibroblasten sein. Bei der Cornea kann die Wirksamkeit mit dem Anteil der enthaltenen lebensfähigen Endothelzellen korreliert sein. Der Wirkmechanismus kann sich hingegen bei Knochen z.B. auf osteokonduktive bzw. osteoinduktive Eigenschaften oder bei anderen GWZ auf deren immunogene Eigenschaften beziehen. Pharmakokinetische Untersuchungen sind für GWZ möglicherweise nicht relevant, sind jedoch in Abhängigkeit der eingesetzten Hilfsstoffe notwendig und müssen für diese plausibel dargestellt werden. Die Toxikologie 
ist hinsichtlich möglicher Abstoßungsreaktionen (Empfänger/Implantat bzw. Implantat/Empfänger), Verunreinigungen (z.B. Reste von Antibiotika oder Freisetzung chemischer Stoffe aus Verpackungsmaterial) oder potentielle Infektionsgefahr durch Viren und bakterielle Erreger zu betrachten.

Das Modul 2.5 soll eine kritische Analyse der vorgelegten klinischen Daten liefern. Die Wirkungen, Nebenwirkungen, Anwendungsgebiete und die Art der Anwendung der GWZ sind durch wissenschaftliches Erkenntnismaterial zu belegen. Erkenntnismaterial für klassische GWZ kann aus Unterlagen über Ergebnisse kontrollierter klinischer Prüfungen oder aus anderweitig bei der klinischen Anwendung wissenschaftlich erhobenen und bewerteten Daten bestehen. Hierzu können Studienergebnisse aus eigenen Untersuchungen, Daten aus Veröffentlichungen oder nachträgliche Bewertungen der klinischen Ergebnisse der zu genehmigenden GWZ herangezogen werden. Auch mit vergleichbaren GWZ gewonnene oder vorliegende Daten können verwendet werden. Voraussetzung ist, dass die Be- oder Verarbeitungsverfahren (z.B. mikrobielle Inaktivierungsschritte und Einsatz von Antibiotika), die Verwendung von Hilfsstoffen (z.B. Glycerin bei Knochenmehlen), die medizinische Indikation und die Funktionalität (z.B. bei Knochenmaterial das Einwachsverhalten und die Fähigkeit, osteokonduktiv zu wirken) vergleichbar bzw. gleichwertig sind. Das Nutzen-Risiko-Verhältnis der GWZ für den zu behandelnden Patienten bzw. die Patientengruppe ist in $\mathrm{Zu}-$ sammenhang mit der festgestellten Funktionalität und Unbedenklichkeit zu diskutieren.

Im letzten Teil (Module 2.6 und 2.7) wird eine Übersicht der relevanten nichtklinischen (diskutiert in Modul 2.4) und klinischen (diskutiert in Modul 2.5) Untersuchungen und Ergebnisse sowohl aus eigenen Studien als auch aus anderem wissenschaftlichem Erkenntnismaterial tabellarisch abgefragt. Die Tabellen werden exemplarisch in den Modulen 2.6 und 2.7 vorgegeben, können jedoch in Abhängigkeit des Datenmaterials angepasst werden. Dies dient der Übersicht über die nichtklinischen und klinischen Untersuchungen, deren Daten und Ergebnisse im einzelnen in den nachfolgenden Antragsmodulen beigefügt werden sollen.

\section{G-CTD-Modul 3: Qualität}

Modul 3 des Genehmigungsantrags soll eine detaillierte Darstellung von Informationen zum Ausgangsmaterial, zum Herstellungsprozess, zu den Qualitätskontrollen bei der Entnahme, Verarbeitung und dem Endprodukt, zur Haltbarkeit und zur Zusammensetzung sowie zur Darreichungsform der beantragten GWZ enthalten. Das Modul ist in zwei Teile gegliedert, die sich auf den Wirkstoff (S; drug substance) und das Arzneimittel (P; drug product) beziehen. Bei muskuloskelettalen GWZ, z.B. Knochenmehl, kann der Wirkstoff das Knochenmehl sein, während das zur medizinischen Anwendung vorgesehene Arzneimittel das Knochenmehl inklusive Hilfsstoff Glycerin sein könnte. Bei Knochenmaterialien, die im Rahmen des chirurgischen Eingriffs erst in der Form und
Größe manuell angepasst werden, können Wirkstoff und Arzneimittel identisch sein. Dies ist der Fall, wenn das zur Anwendung vorgesehene, vollständig bearbeitete Knochenstück in der Zusammensetzung nicht weiter verändert oder mit Zusätzen versehen wird. Ein weiteres Beispiel für die Identität von Wirkstoff und Arzneimittel ist die Cornea, die strukturell unverändert und ohne Zusätze oder Formulierung für die Transplantation verwendet wird.

Die Fragen zum Wirkstoff beziehen sich z.B. bei muskuloskelettalen GWZ auf allgemeine Angaben wie die Bezeichnung des Wirkstoffs (z.B. Knochenmaterial), die Struktur (z.B. die Länge und Gewicht des Knochens), die Beschreibung des Herstellungsprozesses und der entsprechenden Prozesskontrollen sowie auf Fragen zur Haltbarkeit und Stabilität. Des Weiteren werden die relevanten Angaben zu den Ein- und Ausschlusskriterien für die Gewebespender in diesem Modul abgefragt. Nähere Erläuterungen und Beispiele zu den einzelnen Stichpunkten befinden sich im Antragsformular von G-CTD-Modul 3.

\section{G-CTD-Modul 4: Nichtklinische Daten}

Modul 4 dient der Vorlage der Originaldaten und -ergebnisse nichtklinischer Studien bzw. deren plausibler und für die Bewertung hilfreicher Darstellung in übersichtlicher Form. Auch wissenschaftliche Publikationen eigener oder fremder Ergebnisse mit der zur Genehmigung vorgesehenen oder einer gleichwertigen GWZ können hier eingefügt werden. Alle hier aufgeführten Ergebnisse sind in geeigneter Form im G-CTDModul 2 aufzubereiten und zu diskutieren. Neue Informationen sollen nicht eingefügt werden.

\section{G-CTD-Modul 5: Klinische Daten}

Modul 5 dient der Darstellung bzw. Präsentation relevanter klinischer Daten. Das eingereichte Material kann aus nach wissenschaftlichen Methoden aufbereitetem medizinischem Erfahrungsmaterial wie z.B. Studien des Herstellers, Daten aus Veröffentlichungen oder nachträglichen Bewertungen klinischer Ergebnisse bei der Anwendung der GWZ bestehen. Die in G-CTD-Modul 5 abgebildeten Daten sollen bereits in geeigneter Weise in G-CTD-Modul 2 zusammengefasst und diskutiert worden sein. Dieser Abschnitt soll lediglich die in G-CTD-Modul 2 erfolgte Diskussion und Bewertung des Arzneimittels anhand des hier beigebrachten Datenmaterials zur klinischen Entwicklungsstrategie (Studiendesign), Pharmakologie, Wirksamkeit bzw. Funktionalität, Sicherheit sowie zur Nutzen-Risiko-Bewertung belegen.

\section{Vorgehensweise bei der Bearbeitung der Genehmi- gungsanträge gemäß § 21a AMG}

Bis auf wenige Ausnahmen enthielten die bis zum 1. Februar 2008 beim Paul-Ehrlich-Institut eingegangenen Anträge auf Genehmigung von GWZ gemäß $§ 21 \mathrm{a} A M G$ nicht die für eine
460

Transfus Med Hemother 2008;35:453-462
Schilling-Leiß/Godehardt/Scherer/

Cichutek/Tönjes 
Beurteilung ausreichenden Unterlagen. Um die Antragsteller in dieser Situation zu unterstützen und um eine Verknappung der klassischen GWZ zu vermeiden, wählte das Paul-EhrlichInstitut eine angemessene Vorgehensweise. Zur Behebung der Antragsmängel hat das Paul-Ehrlich-Institut bei unvollständigen Anträgen den Antragstellern eine Frist von 6 Monaten gesetzt. Für den Zeitraum bis zur Behebung der Antragsmängel wird die gemäß $\S 21 \mathrm{a}$ Abs. 4 AMG vorgesehene Frist von 5 Monaten für die Genehmigung gemäß § 21a Abs. 1 AMG gehemmt. Eine schnellere Behebung der Mängel durch den Antragsteller verkürzt den Zeitraum der Fristhemmung und reduziert folglich die Zeit bis zu einer Entscheidung über den Genehmigungsantrag.

Gleichzeitig bestand jedoch Mitte 2008 die Situation, dass viele eingegangene Anträge für die Genehmigung einer klassischen GWZ gemäß $§ 21$ a Abs. 1 AMG wegen der genannten Antragsmängel keine Entscheidung darüber erlaubten, ob statt eines Genehmigungsantrags gemäß § 21a Abs. 1 AMG nicht ein Zulassungsantrag gemäß § 21 Abs. 1 AMG zu stellen war. Dies wäre dann der Fall, wenn die in § 21a AMG genannten Bedingungen für die Antragstellung nicht gegeben wären. In solchen Fällen war das Paul-Ehrlich-Institut bestrebt, den Antragstellern durch eine frühzeitige Entscheidung über die Zulässigkeit des Genehmigungsantrags gemäß $\S 21 \mathrm{a}$ AMG Gelegenheit zu geben, noch zum Stichtag 30. September 2008 einen Zulassungsantrag gemäß $§ 21$ Abs. 1 AMG zu stellen und damit bereits im Verkehr befindliche GWZ weiter in Verkehr bringen zu dürfen, bis über den Antrag entschieden wurde. Dazu wurden die Antragsteller im Rahmen der Behebung von formalen Antragsmängeln aufgefordert, bereits einige Wochen vor dem 30. September 2008 Angaben über das Vorliegen der Voraussetzungen für die $\mathrm{Zu}-$ lässigkeit eines Genehmigungsantrags gemäß § 21a Abs. 1 AMG zu machen.

Des Weiteren soll dem Antragsteller ermöglicht werden, auf bereits vorliegende geprüfte und freigegebene Antragsteile Bezug zu nehmen. Voraussetzung hierfür ist, dass die entsprechenden Datenangaben für die in Frage stehende Genehmigung der GWZ ohne Ausnahme gelten. Durch die Gruppierung der GWZ (Abb.1) und die Bereitstellung von Musteranträgen, z.B. für vergleichbare Augenhornhäute oder muskuloskelettale GWZ, wird das Verfahren der Genehmigung gemäß $\S 21$ a AMG wirkungsvoll vereinfacht und der Aufwand für die Erstellung, Bearbeitung und Prüfung identischer oder ähnlicher Produkte auf ein Minimum begrenzt. Der zügigen Antragsbearbeitung diente ebenfalls der Vorschlag des Paul-Ehrlich-Instituts, vom Musterantrag abweichende Passagen des jeweiligen Genehmigungsantrags deutlich hervorzuheben.

Die Genehmigung der GWZ gemäß § 21a Abs. 1 AMG erfolgt nach Bewertung durch das Paul-Ehrlich-Institut, wenn die Antragsunterlagen vollständig vorgelegt werden, die GWZ dem Stand der wissenschaftlichen Erkenntnisse entspricht, ihre Funktionalität dargelegt wurde und das NutzenRisiko-Verhältnis günstig ist.

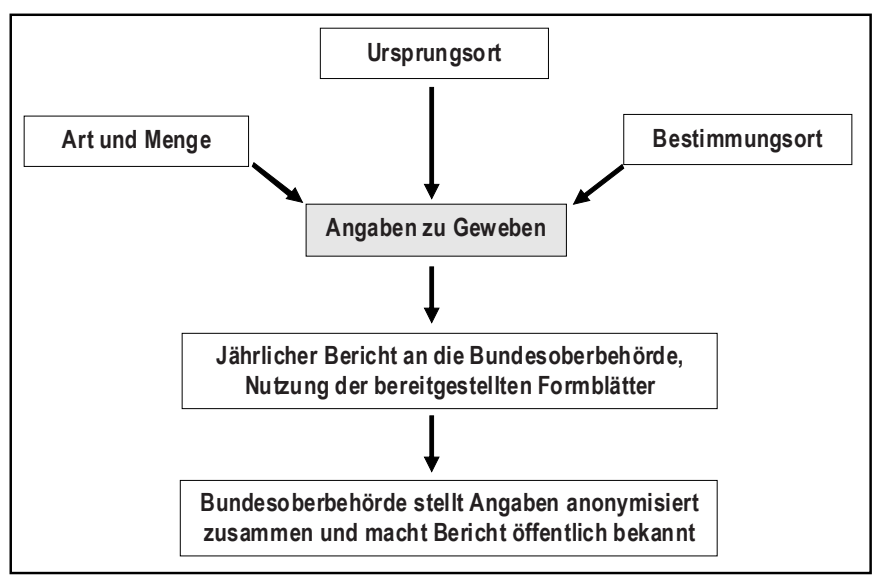

Abb. 3. Schematische Übersicht zur Dokumentation der Tätigkeit von Gewebeeinrichtungen gemäß $\S 8 \mathrm{~d}$ Abs. 3 TPG.

\section{Besondere Dokumentations- und Meldepflichten bei Gewebezubereitungen}

Genehmigungs- und Zulassungsinhaber, aber auch Antragsteller zum Stichtag 1. Februar 2008 für eine Genehmigung gemäß § 21a Abs. 1 oder Abs. 9 AMG und Antragsteller zum Stichtag 30. August 2008 für eine Zulassung gemäß $§ 21$ Abs. 1 AMG sowie Entnahmeeinrichtungen und Gewebespenderlabore haben unter anderem die Verpflichtung gemäß $\S 63 \mathrm{c}$ Abs. 2 AMG, jeden Verdacht eines schwerwiegenden Zwischenfalls, der sich auf die Qualität oder Sicherheit einer GWZ auswirken kann, und jeden Verdacht einer schwerwiegenden unerwünschten Reaktion, die die Qualität oder Sicherheit der GWZ beeinflussen oder auf sie zurückgeführt werden kann, unverzüglich dem Paul-Ehrlich-Institut anzuzeigen. Gemäß § 40 AMWHV ist die verantwortliche Person nach § 20c AMG dafür verantwortlich, die entsprechenden Meldungen zu sammeln, zu bewerten und an die zuständige Behörde zu übermitteln. Formblätter für derartige Anzeigen werden auf der Internetseite des Paul-Ehrlich-Instituts zur Verfügung gestellt. Verdachtsfälle schwerwiegender Zwischenfälle, z.B. in Bezug auf die mikrobielle und die Virussicherheit, können erst nach der Freigabe einer produzierten GWZ bzw. bei mangelhaften Chargen erhoben werden. Verdachtsfälle einer oben genannten schwerwiegenden unerwünschten Reaktion sind z.B. Erregerübertragungen, die in Zusammenhang mit der Übertragung einer GWZ bei einem oder mehreren Empfängern auftraten.

\section{Meldepflicht der Gewebeeinrichtungen gemäß § 8d Abs. 3 TPG}

In $\S 8 \mathrm{~d}$ TPG sind weitere besondere Pflichten für Gewebeeinrichtungen festgelegt (Abb. 3). So muss eine Gewebeeinrichtung gemäß $\S 8 \mathrm{~d}$ Abs. 3 TPG dem Paul-Ehrlich-Institut jähr- 
lich einen Bericht vorlegen, der Art und Menge der entnommenen, aufbereiteten, be- oder verarbeiteten, aufbewahrten, abgegebenen oder anderweitig verwendeten, eingeführten und ausgeführten Gewebe darlegt. Das Paul-Ehrlich-Institut hat für diese Meldung ein Formblatt entworfen und im Bundesanzeiger sowie auf seiner Internetseite bekannt gemacht (www.pei.de/cln_047/nn_160656/DE/infos/pu/08-meldepflichten/doku-tpg8/doku-tpg8-node.html?_nnn=true). Der jährliche Meldebogen der Gewebeeinrichtung ist spätestens bis zum 1. März des Folgejahres an das Paul-Ehrlich-Institut zu übermitteln. Bei fehlenden Berichten ist das Paul-Ehrlich-Institut verpflichtet, die zuständige Behörde zu unterrichten. Die Gewebeeinrichtungen übersenden der zuständigen Landesbehörde alle 2 Jahre oder auf Anforderung eine Liste der belieferten Einrichtungen der medizinischen Versorgung.

Aus den anonymisierten Angaben der Einzelberichte der Gewebeeinrichtungen erstellt das Paul-Ehrlich-Institut einen Gesamtbericht, der veröffentlicht wird. Die Bundesregierung hat nach 3 Jahren und im weiteren alle 4 Jahre einen Erfahrungsbericht zu den Auswirkungen des Gewebegesetzes zu veröffentlichen. Das Paul-Ehrlich-Institut wird hierzu seine Erfahrungen aus den Genehmigungsverfahren sowie die Auswertung der jährlichen Meldungen der Gewebeeinrichtungen beitragen. Die zunächst schriftlichen Meldungen für das Jahr 2007 sollten nur den Zeitraum ab Inkrafttreten des Gewebegesetzes, 1. August bis 31. Dezember 2007, erfassen. Es ist mittelfristig vorgesehen, ein elektronisches Meldesystem zu etablieren, um das Verfahren zukünftig effizienter zu gestalten.

Da menschliche Keimzellen (Spermien, Eizellen) und Embryonen gemäß $§ 4$ Abs. 30 AMG Gewebe darstellen, aber nicht GWZ und damit auch keine Arzneimittel sind, ist in diesen Fällen keine Genehmigung nach § 21a AMG erforderlich. Dennoch müssen von reproduktionsmedizinisch tätigen Gewebeeinrichtungen jährlich Meldebögen erstellt werden. Spezielle Erläuterungen zur Meldung von Keimzellen werden durch das Paul-Ehrlich-Institut und durch den Bundesverband Reproduktionsmedizinischer Zentren Deutschlands bereitgestellt.

\section{Literatur}

1 Beschlussempfehlung und Bericht des Ausschusses für Gesundheit (14. Ausschuss) zu dem Gesetzesentwurf der Bundesregierung. 2007. Bundesdrucksache 16/5443. 23.05.2007. S. $1-60$

2 Antwort der Bundesregierung auf die Kleine Anfrage der Abgeordneten Dr. Haralf Terpe, Birgitt Bender, Elisabeth Scharfenberg, weiterer Abgeordneter und der Fraktion BÜNDNIS 90/DIE GRÜNEN. 2008. Bundesdrucksache 16/9988. 15.07.2008. S. 1-10. 